\title{
Aportes en torno a la desjudicialización de la identidad de género de niños, niñas y adolescentes
}

por Aramis Lascano

\begin{abstract}
Resumen
En primer lugar, introduciré algunas cuestiones y categorías que atraviesan el desarrollo del presente trabajo, advirtiendo el impacto de la sanción de la "Convención de los Derechos del Niño" en la mirada sobre la multiplicidad de problemáticas que involucran a niños, niñas y adolescentes. En segundo lugar, realizaré una caracterización breve sobre la situación del colectivo trans-travesti y la legislación vigente, para luego desarrollar la situación judicial elegida mencionando tanto la plataforma fáctica como la ruta atravesada por la joven trans, su madre y los/as profesionales que la acompañaron hacia la judicialización, y posterior sentencia. Por otro lado, a partir de una mirada crítica del 'corpus' del fallo, aportaré algunos elementos y caminos posibles a la decisión judicial finalmente tomada para, finalmente, añadir algunas palabras finales hacia la desjudicialización de la identidad de género.
\end{abstract}

Palabras clave

Género, derechos humanos, transgénero, identidad

\section{Summary}

First of all, I will introduce some questions and categories which crossed the development from this paper, emphasizing the impact which caused the "Convention on the Rights of the Child" on the point of view about great variety childs and adolescents problems. In second place, I will make a little description about the reality of transgender people and the current law to develop the precedent chosen, describing the fact as well as the experience lived by the transgender adolescent, her mother and the professionals who worked with them until the sentence. At the end, I will analyze the essential point from the sentence, I will contribute some elements and alternatives to avoid the judicialize the gender identity.

Key words

Gender, human rights, transgender, identity 


\section{Algunas aclaraciones fundamentales}

Una de las instituciones más cuestionadas por los feminismos del siglo XX fue, indudablemente, la familia. En particular, las críticas se dirigieron a la concepción imperante desde el siglo XIX de la familia nuclear como un "orden natural" que articula una sexualidad "natural" ta heterosexual y monógama-, una reproducción "natural" -la heterosexual y con la crianza a cargo de las mujeres-, una producción "natural" -por fuera del hogar y a cargo de los varones-, y una disciplina "natural" -la de los varones sobre las mujeres y los/as niños/as y de las mujeres sobre los/as niños/as- (Jaramillo, 2008: 267).

En este sentido, opto por hablar de heteropatriarcado (y no de patriarcado y heteronormatividad en forma escindida) no solo para subrayar a ambos componentes como elementos coconstitutivos sino para explicitar y nombrar el rol fundamental de la heteronormatividad como "principio organizador del orden de relaciones sociales, política, institucional y culturalmente reproducido, que hace de la heterosexualidad reproductiva el parámetro desde el cual juzgar (aceptar, condenar) la inmensa variedad de prácticas, identidades y relaciones sexuales, afectivas y amorosas existentes" (Pecheny,2008:14). La heteronormatividad sirve como matriz para la estigmatización, exclusión, invisibilización y criminalización de las identidades disidentes (gays, lesbianas, travestis, transexuales, transgéneros, bisexuales, queer e intersexuales) y de vínculos en oposición al patrón heterosexual. Es así que el sistema heteropatriarcal funciona "como un sistema de regulación de cuerpos y sexualidades, y de ordenamiento de esferas socioeconómicas, que permite la existencia de ámbitos feminizados al servicio de los masculinizados y garantiza la existencia de sujetos subalternos que los ocupen" (Orozco, La Fuente, 2014:102) en el marco del capitalismo globalizado.

En particular a la situación de las/os niñas/os -o las niñeces ${ }^{1}$-, cabe agregarle al componente heteropatriarcal de la sociedad, lo adultocéntrico; esto es, aquella matriz que "pone en condición de inferioridad y de 'preparación hacia' a niñas, niños y jóvenes, y a las y los adultos mayores como 'saliendo de"” (Duarte, 2006:12). La mirada adultista funciona como

1 El Colectivo "Piedra, Papel y Tijera" es una organización social y política que trabaja hace varios años con niños, niñas y adolescentes de barrios populares, en particular, del Barrio "El Carmen" de la ciudad de Berisso. Aprendí junto a ellas/os, que, etimológicamente, la palabra infancia -que proviene del latín-, significa "el que no tiene la capacidad del habla". Y, por otro lado, no creo conveniente utilizar la categoría "niñez" sino optar por "niñeces" buscando problematizar aquellas miradas que homogeneizan dicha población, al mismo tiempo que la normalizan, negando, así, los atravesamientos de clases, géneros, etnias, etc., que luego mencionaremos. 
parámetro para el mundo juvenil, impone un 'deber ser', lo que debe hacerse para ser considerado en la sociedad (madurez, responsabilidad, integración al mercado de consumo y de producción, reproducción de la familia, participación cívica, etc.) (Ibíd:65).

Las concepciones dominantes en el derecho no escapan a la presencia de estas matrices -heteropatriarcales, adultistas, capitalistas-, sino que inclusive se encargan de trabajar en su reforzamiento permanente a partir de, por ejemplo, pensar al varón blanco, cisheterosexual ${ }^{2}$, blanco, de clase media, adulto, sin discapacidades como paradigma de sujeto de derecho.

La perspectiva adultista de observar al mundo, afectó claramente al campo de estudios sobre las niñeces, si tenemos en cuenta que hasta no configurar una cuestión social, los abordajes fueron escasos y es recién a partir de la década del '90 en que empiezan a desarrollarse en volumen investigaciones y trabajos de campo. El impacto en la academia nacional y latinoamericana fue acelerado por la realización de la "Cumbre Mundial de la infancia" en Nueva York en 1990, en la cual Argentina lleva como compromiso la ratificación de la "Convención de los Derechos del Niño" (en adelante, CDN) e instala la necesidad de implementar programas y acciones destinados a ella, así como deja en evidencia los niveles de ignorancia existentes sobre la temática (Güerureman, Macri, 2013:134).

$\mathrm{Si}$ bien existieron algunos instrumentos normativos internacionales sancionados previamente a la CDN en $1989^{3}$ y el concepto de los/as niños/as como sujetos de derecho internacional es anterior a ella, esta convención introduce numerosos estándares de derechos humanos y es un programa de acción para los Estados y las sociedades que la han ratificado:

no es enunciado de buenos propósitos. Implica transformaciones en las relaciones del niñx con el Estado, la familia y la comunidad, por lo que las condiciones de posibilidad para hacer efectivos los derechos allí enunciados descansan en cambios en la normativa jurídica que regula dicha relaciones, las estructuras institucionales y las prácticas sociales en las que interactúan adultos y niñxs juntos (Pérez Cazenave, 2017:343).

No es irrelevante mencionar el momento histórico y las circunstancias sociopolíticas en la cual fue ratificada e incorporada la CDN por países de América Latina -en nuestro país, tiene jerarquía constitucional, según el art. 75, inc. 22 de la Constitución Nacional-y por qué el enfoque dominante

2 Cis (Lat., "de este lado") es un concepto que se construyó en oposición a lo "trans" (Lat., "del otro lado") y que, en síntesis, se lo utiliza para referirse a aquella persona cuya identidad de género coincide con el sexo que le fue asignado al nacer.

3 Como la Declaración de Ginebra en 1924, adoptada por la Unión Internacional para la Protección de la Infancia. 
puso el énfasis en las libertades negativas: "éste presupone un modelo de Estado que tiende a reducirse a su mínima expresión, Estado-gendarme eventualmente eficiente pero no preocupado por las prestaciones positivas características del Estado de Bienestar en crisis que se pretendía transformar" (Beloff, 2011:2).

De esta manera, la CDN vino a sustituir al "tutelarismo clásico", expresión que alude al conjunto de teorías y dispositivos que estructuraban a la justicia de menores o los sistemas tutelares administrativos de menores latinoamericanos (Ibíd., 6) y que, en líneas generales, se basaba en una mirada paternalista que piensa a los/as niños/as y adolescentes como objetos de protección y custodia -sometidos a la intervención autoritaria del Estado y, sobre todo, del Poder Judicial- y no como sujetos/as de derechos. Es así que, a partir de la CDN y, en consecuencia, de la sanción de la Ley 26.061 y la Ley 13.298, en el ámbito de la Nación y de la provincia de Buenos Aires, respectivamente, se planteó este nuevo paradigma de promoción y protección de derechos según el cual, todo lo que disponga el Estado, deberá ser atendiendo al "interés superior del niño" y a la salvaguarda de sus derechos cuestiones que luego profundizaré-.

\section{Las niñeces más allá del binario}

A diferencia de otros grupos sociales desaventajados, la familia suele ser el primer obstáculo que enfrentan las travestis, transexuales o transgéneros para el libre desarrollo de su identidad. En general, hay una situación inicial de desprotección y expulsión del hogar, aunque la realidad y los vínculos familiares se matizan de acuerdo a distintos factores como la edad en que se asume la identidad de género, la región en la que habitan, etc.,(Berkins, 2008: 68-70).

La Ley de Identidad de Género (Ley 26.743; en adelante, LIG) fue sancionada en la Cámara de Diputados/as -por unanimidad- en mayo de 2012, producto de la tenaz lucha y articulación del movimiento LGBTTTIQ $^{4}$, en particular, del movimiento trans y travesti encolumnado en el Frente Nacional por la Identidad de Género. Esta legislación, vanguardia en el mundo, está anclada bajo los principios de la autodeterminación, desjudicialización y despatologización de las identidades y corporalidades trans y travestis: garantiza el acceso al cambio registral de nombre y sexo mediante un 'simple' trámite administrativo y es la única que garantiza el acceso a procedimientos de 'afirmación de sexo' con el único requisito del

4 Sigla que comprende a lesbianas, gays, bisexuales, travestis, transexuales, transgénero, intersex y queer. 
consentimiento informado de la persona requirente, y con la cobertura del Plan Médico Obligatorio.

Ya no es necesario someterse a diagnósticos o dictámenes biomédicos y/o resoluciones judiciales condicionales -salvo alguna excepción legal, como luego mencionaré- para ejercer el derecho a la identidad de género y a la ciudadanía. La LIG deja en estado de indeterminación una de las entidades más sólidas del imaginario social: la dupla dicotómica "masculinofemenino", entendida como una colección contigua de hechos biológicos, configuraciones psíquicas, modalidades expresivas, roles adscriptos de funcionalidad y apariencias para la interacción social (Meccia,2012).

Más allá de los avances formales en materia de derechos (sobre todo, a partir de la sanción en 2012 de la LIG), la situación de la inmensa mayoría de las personas travestis, transexuales y transgéneros en la Argentina es, problemática ya sea desde el acceso a la ciudadanía y/o en el campo de la salud, la educación y el trabajo, conviviendo y enfrentándose con distintas clases de violencias (estatal, sexual, doméstica, etc.,) (Berkins, 2013: 6).

\section{Judicializando la identidad de género}

La situación elegida para el presente trabajo aborda la trayectoria judicial de J., una joven transgénero de 15 años de edad ${ }^{5}$, oriunda de una ciudad del interior de la provincia de Buenos Aires. J. comenzó a exteriorizar su identidad de género en la escuela en el año 2009 cuando rondaba los 7 años de edad-aunque ya dos años atrás comenzaba a expresarse con otro género al asignado al nacer-.

Desde la dirección del colegio al que concurría, manifestaron que "no estaban preparados para estos casos", y la enviaron a realizarse estudios psiquiátricos, psicológicos y genéticos. Sugirieron que su madre estaba ejerciendo "una influencia negativa" y hasta circuló la delirante hipótesis de que, como vive en un pueblo rural de la provincia de Buenos Aires, los agroquímicos podrían ser los causantes de esos 'efectos indeseados'(Curia, 2016). Las respuestas desde los/as profesionales fue sugerir el disciplinamiento: había que corregir los comportamientos de J.

5 Si bien el texto de la sentencia fue difundido, ésto sucedió sin autorización de la joven trans, su madre y/o los/as abogados/as que intervinieron, quienes habían requerido que se resguarde la intimidad e integridad de la adolescente al juzgado interviniente. La información para el desarrollo del presente trabajo fue recabada mediante conversaciones con un integrante del colectivo ABOSEX, el abordaje periodístico, parte del texto de la sentencia y complementada con la publicación en la página de esta organización, que destaca aspectos relevantes del fallo. 
La expulsión escolar, la patologización, la responsabilización de la 'madre' y la represión interdisciplinaria son parte del cóctel habitual que suelen rondar en torno al abordaje de las identidades trans y travestis. Novedosa fue la incorporación de los agroquímicos como condimento 'causal de la desviación': las personas transgénero nuevamente eran incorporadas al capítulo de la "teratología", esa disciplina encargada del tratamiento de los seres humanos sexualmente monstruosos e inexplicables (Ben, 2000).

La situación que estaba atravesando J. llegó a la organización Mamichula, que contactó a J. y su madre con Abogadxs por los derechos sexuales (ABOSEX) en el año 2010. La actuación de este colectivo permitió que J. pudiese reincorporarse al colegio, y su madre, luego de interiorizarse y comprender las vivencias de su hija, comenzó a apoyarla y la autorizó para la modificación registral. Sin embargo, su padre -que no convive con ellas y prácticamente no tenía contacto- no prestó su consentimiento en orden a la rectificación registral que se pretende, conforme el procedimiento de la ley de identidad de género. Por ello, teniendo en consideración lo dispuesto por el art. 5 de la LIG -que luego analizaré-, hubo que acudir, en el año 2015, a la judicialización de la situación de J.

Conforme al art. 27 de la Ley de Protección Integral de los Derechos de las Niñas, Niños y Adolescentes (Ley 26.061, 2005) y la Ley 14.568 cumpliendo con lo establecido por el artículo 12 , incs. 1 y 2 de la CDN y el artículo $8^{\circ}$ del Pacto de San José de Costa Rica-, se le designó una abogada a la joven para que represente técnicamente sus intereses, distinto al de su madre en función de los derechos personalísimos en disputa.

Desde la parte accionante, se sostuvo que en la partida de nacimiento de la niña estaba consignado el sexo masculino y nombre de varón; que la niña se identificada desde los 5 años con el "sexo femenino", y que actualmente concurría a la escuela y a otras actividades conforme a su identidad de género autopercibida, que expresa en forma sostenida y libre. Se sostuvo que su documentación registral no concuerda con su identidad de género, lo que facilitaba tratos discriminatorios y ponía en riesgo 'el desarrollo de una vida plena y libre de violencia' y que, el pedido era una decisión de la niña, luego del asesoramiento recibido en un lenguaje accesible y adecuado a su madurez.

Finalmente, luego de la intervención del agente fiscal y el asesor de incapaces -quien entiende que se debe hacer lugar a lo solicitado por la accionante-, J. fue escuchada por la jueza en audiencia que se celebrara con la presencia de la perito psicóloga del juzgado de familia, la secretaria de la Asesoría de Incapaces, y su abogada. Resulta interesante recuperar de la sentencia, parte de la apreciación judicial del testimonio de la joven trans: 
...que tiene 14 años de edad, que tiene conocimiento sobre los motivos de la audiencia, hizo saber que concurre a la Escuela [...] cursando $6^{\circ}$ grado del ciclo primario por la mañana, que antes cursaba en la Escuela [...]; expuso que viajaba a la ciudad de La Plata y era atendida por un psicólogo. Sostuvo que en relación a sus compañeros de clase no tiene ningún problema en lo que hace a su condición de género, aunque no tiene demasiada relación con ellos. Que vive junto a sus dos hermanos B. y K.A y el señor M. A quien es la pareja de su madre. Dio cuenta que fue por motivos propios que le solicitó a su madre el cambio de identidad en el documento, que con su padre mantiene escaso contacto y cuando le propuso el cambio de género, éste no le contestó, cambió el tema. Expuso estar al tanto de la ley de identidad de género. Que siempre la mandaron de la escuela a realizar tratamiento psicológico pero que no quiere hacerlo por propia voluntad (Consid. $5^{\circ}$ ).

La joven también manifestó que:

siempre la mandaron de la escuela a realizar tratamiento psicológico pero que no quiere hacerlo por propia voluntad. Que viaja a Buenos Aires donde es sometida a un tratamiento. Refiere que quiere agregar a su nombre el de I., quedando formado el mismo como I. N.J. Que, en el Hospital Pedro de Elizalde en la ciudad de Buenos Aires, comenzará un tratamiento psicológico, donde realiza un tratamiento hormonal y que en la Escuela es atendida por el Gabinete Pedagógico (Ibíd.).

Por otro lado, del informe de la perito psicóloga, trasciende que:

su decisión de cambiar su documento de identidad, surge a partir de amigos y amigas transexuales que tiene vía facebook. Que allí supo de esa posibilidad, y decidió hablar con su madre para iniciar el trámite. $\mathrm{Su}$ padre no quiso firmar el consentimiento, entendiendo que la presente instancia tiene lugar por esa razón. N. manifiesta que en la actualidad no tiene ningún inconveniente con sus compañeros en el ámbito escolar. Aclara que se atrasó dos años por dificultades surgidas en la institución donde concurría antes, a partir de su elección de género. Sus amistades más cercanas pertenecen a su barrio y son con quienes se visita habitualmente y comparte distintos momentos. Comenta que concurría a danzas árabes pero que al momento no lo hace. (Ibíd.)

La perito psicóloga concluye que:

al momento de la audiencia $\mathrm{N}$. se presenta lúcida globalmente orientada y con las funciones de su psiquismo conservadas. No se advierten signos de psicopatología de mención ${ }^{6}$. Es clara cuando se

6 No fue posible con los recursos disponibles para la realización del presente trabajo, determinar la alusión a la carencia de una psicopatología. 
manifiesta respecto de su elección de género. Está al tanto de la ley que la ampara. Expresa su deseo de agregar un tercer nombre al ya elegido I. N.J para que conste en su DNI. Inserta en un espacio familiar en el que se siente contenida y escuchada. Se infiere que se desarrolla en un ámbito propicio y acorde a su edad. (Ibíd.)

Cabe agregar que el padre de la joven nunca se presentó en el proceso pese a estar notificado fehacientemente. Solo obstaculizó la decisión de su hija, lo cual tuvo como consecuencia la judicialización del ejercicio del derecho a la identidad.

La jueza sentencia concediendo la autorización peticionada, ordenando la rectificación de la partida de nacimiento correspondiente y la expedición de un nuevo documento nacional de identidad, tal como lo estipula el artículo 4 de la LIG. Los principales argumentos utilizados por la jueza se centraron en: el interés superior de la niña, la capacidad progresiva y lo dispuesto por la LIG.

En referencia al interés superior, expresó que este principio, plasmado en el art. 3 de la CDN, reconocido en diversas leyes infraconstitucionales y en el Código Civil y Comercial de la Nación (CCyCN):

sirve de guía al juzgador y condiciona cualquier solución en aquellos conflictos en que existan intereses contrapuestos. El niño como sujeto activo y autónomo tiene derecho a una protección especial que debe prevalecer como factor esencial, determinando que su interés moral y material debe tener prioridad por sobre cualquier otra circunstancia $\left(\right.$ Consid. $1^{\circ}$ ).

Asimismo, tiene en consideración la Observación General N 14 emitida por el Comité de los Derechos del Niño en 2013, que señala que este interés superior tiene tres conceptos: como derecho sustantivo, esto es, como derecho de la niña/o a que interés superior sea una consideración primordial ponderable y tenido en cuenta al sopesar distintos intereses como, en este caso, el del padre- para tomar una decisión; como principio jurídico interpretativo fundamental, es decir, se jerarquizará la interpretación que satisfaga de manera más efectiva el interés superior de la niña/o; y como norma de procedimiento, esto es, que deberá tenerse en cuenta, siempre que se tome una decisión que afecte a niños/as y adolescentes, el impacto que puede tener en éstas/os.

Por su parte, con respecto a la capacidad progresiva de la niña, entiende a ésta como parte del cambio de paradigma que surge de la CDN, y en línea con lo dispuesto por el art. 5 de la convención citada y el art. 26 del $\mathrm{CCyCN}$, sostiene que la capacidad "no está sujeta a categorías fijas, sino que es un sistema progresivo de autonomía que no tiene sujeción a una edad 
cronológica, sino que opera en función de la madurez intelectual y psicológica y grado de desarrollo del niño" (Consid. $2^{\circ}$ ). Argumenta que la noción de autonomía progresiva en el ejercicio de los derechos traslada "el eje de la mirada desde el concepto rígido de capacidad hacia la noción de origen bioético de competencia. Esta habilita la actuación de derechos en forma directa por su titular" (Ibíd.) aun cuando no ostente plena capacidad civil y, teniendo en cuenta, la ponderación de que la persona pueda formar convicción y decisión razonada respecto de la cuestión que la involucra.

Finalmente, en cuanto a la mención a la LIG, en primer lugar, menciona lo dispuesto por los arts. $1^{7}$ y $2^{8}$, en cuanto a qué implica el derecho a la identidad de género y qué se entiende por éste. Para posicionarse respecto a la noción de género, sexo e identidad comienza a realizar algunas citas de doctrina jurídica ${ }^{9}$. En primer lugar, con respecto a la escisión entre género e identidad, expresa que:

Género e identidad no son aspectos opuestos, sino que se complementan. La identidad constituye un concepto multifacético; está ligada a la noción de permanencia. El derecho a la identidad no se limita a considerar el aspecto físico o biológico de la persona; comprende también el bagaje espiritual, intelectual, político, profesional, a través del cual, el individuo se proyecta socialmente exteriorizando su personalidad (Ciolli, 2012:11 en Consid. $4^{\circ}$ ).

Por otro lado, para definir qué entiende por género, acude a otra cita de doctrina jurídica:

el género se define como un conjunto de normas de comportamiento percibidas particularmente como masculinas o femeninas. Cuando el

7 Art. 1: Derecho a la identidad de género. Toda persona tiene derecho: a) Al reconocimiento de su identidad de género; b) Al libre desarrollo de su persona conforme a su identidad de género; c) A ser tratada de acuerdo con su identidad de género y, en particular, a ser identificada de ese modo en los instrumentos que acreditan su identidad respecto de el/los nombre/s de pila, imagen y sexo con los que allí es registrada.

8 Art. 2: Definición. Se entiende por identidad de género a la vivencia interna e individual del género tal como cada persona la siente, la cual puede corresponder o no con el sexo asignado al momento del nacimiento, incluyendo la vivencia personal del cuerpo. Esto puede involucrar la modificación de la apariencia o la función corporal a través de medios farmacológicos, quirúrgicos o de otra índole, siempre que ello sea libremente escogido. También incluye otras expresiones de género, como la vestimenta, el modo de hablar y los modales.

9 Si bien se puede compartir o no lo sostenido por esta autora -por lo menos, resulta discutible la necesaria e indisoluble vinculación entre identidad y permanencia, sin mayores precisiones-, es interesante analizar que ésta es una de las pocas citas doctrinarias en la sentencia, que proviene de una revista jurídica -y una autora abogada-, y no de una publicación u autora/r del campo de los estudios de género o queer, lo cual permite que se indique cierto enjaulamiento disciplinario en el marco de la sobrejerarquización del derecho como saber - situado sobre el resto de los saberes y disciplinas- 
género asignado al nacer no se corresponde con el género con el que uno se identifica la persona sufre muchísimos problemas, sociales, económicos, prácticos y de identidad que requieren solución (Medina, 2012:45 en Consid. $\left.4^{\circ}\right)^{10}$.

Luego, prosigue citando a lo dispuesto por los arts. 4 y 5 de la LIG, respecto a las personas menores de edad, y concluye que, el interés superior, la capacidad progresiva, el derecho a ser oída vinculado con el derecho a la identidad de género -y el derecho a la dignidad, libertad-y la condición de sujeto de derecho implica que no puede desconocerse "la realidad humana en que se encuentra [la niña] siendo el Estado quien brinde satisfacción a todos los derechos y garantías mencionados, brindándole una respuesta jurídica adecuada" (Consid. $5^{\circ}$ ).

La falta de presentación del padre de J., el contacto y la escucha mantenida con la niña y su madre, los informes de los/as profesionales, el dictamen favorable del asesor de incapaces, fueron factores trascendentales, para la jueza, a la hora de conceder la autorización peticionada por la parte accionante.

\section{Algunas observaciones a los argumentos}

Ahora bien, el resultado favorable de la petición formulada por J., no quita que se puedan analizar críticamente algunos extractos de la sentencia y la interpretación del articulado de la LIG y de la CDN. Uno de las transformaciones sustanciales aportadas por la LIG es la desjudicialización como principio, esto es, que las personas travestis, transexuales y transgénero que tengan 18 años de edad puedan realizar la rectificación registral sin necesidad de someterse al arbitrio judicial y/o a condicionamientos médicos, siendo necesario un trámite administrativo para modificar los datos personales en la partida de nacimiento y así obtener un nuevo documento nacional de identidad con el nombre autopercibido, que respete la identidad de género.

¿Qué sucede con las personas que no llegan a esa edad preestablecida? En primer lugar, es necesario analizar lo regulado por el artículo 5 de la LIG. Esta disposición determina que:

Con relación a las personas menores de dieciocho (18) años de edad la solicitud del trámite a que refiere el artículo $4^{\circ}$ deberá ser efectuada a

10 Esta autora parece cometer una confusión conceptual -bastante habitual- sobre la noción de "sexo" y "género" -en particular, al reconocer la posibilidad de que la determinación genital y/o biomédica asigne un "género" y no un "sexo"- aunque por el escaso desarrollo no es posible afirmarlo. 
través de sus representantes legales y con expresa conformidad del menor, teniendo en cuenta los principios de capacidad progresiva e interés superior del niño/a de acuerdo a lo estipulado en la Convención sobre los Derechos del Niño y en la Ley 26.061 [...]. Cuando por cualquier causa se niegue o sea imposible obtener el consentimiento de alguno/a de los/as representantes legales del menor de edad, se podrá recurrir a la vía sumarísima para que los/as jueces/zas correspondientes resuelvan, teniendo en cuenta los principios de capacidad progresiva e interés superior del niño/a de acuerdo a lo estipulado en la Convención sobre los Derechos del Niño y en la Ley 26.061 de protección integral de los derechos de niñas, niños y adolescentes (Ley 26.743, 2012).

La desjudicialización como regla se altera cuando uno/a de los/as representantes legales, por cualquier motivo, se niegue o sea imposible obtener su consentimiento. Parece contradictorio que, por un lado, se tenga en cuenta la autonomía progresiva de las niñas/os y adolescentes (art.5, CDN; art. 24, inc. B, Ley 26.061) y, por el otro, se requiera que la solicitud sea requerida mediante sus representantes legales "con expresa conformidad del menor". El principio de autonomía progresiva contempla las diferentes etapas por las que atraviesan los niños/as en su evolución psicofísica, determinando una gradación en el nivel de decisión al que puede acceder en el ejercicio de sus derechos fundamentales; se aparta del concepto de capacidad, y "se condice con la noción más empírica - de origen bioético- de competencia, conformada a partir del desarrollo de una conciencia reflexiva, libre, racional, independientemente de la ubicación del niño conforme el concepto de capacidad civil de rígida determinación" (Fernández, 2012:6). El art. 5 de la LIG estaría en colisión con lo dispuesto por el art. 26 del Código Civil y Comercial de la Nación ${ }^{11}$-vigente al momento de la sentencia- que establece que: "...la persona que cuenta con edad y grado de madurez suficiente puede ejercer por sí los actos que le son permitidos por el ordenamiento jurídico"12 (Ley 26.994, Art.5, 2014).

Por su parte, tanto la CDN, como la Ley 26.061, establecen que la capacidad sería la regla, y la incapacidad la excepción para el ejercicio de los derechos por parte de los niños/as y adolescentes. Y como se advertía, sin estar vigentes aún las disposiciones del Código Civil y Comercial actual:

11 En el artículo 25, se determina que "menor de edad" es toda persona que no haya cumplido los 18 años y que "adolescente" es toda persona menor de edad, que cumplió 13 años de edad.

12 Ante la posibilidad de colisión de derechos entre representantes y niños/as y adolescentes, se establece a continuación la facultad de adolescente de defender su posición con el auxilio de asistencia letrada. 
se encontraría invertida la carga probatoria o el onus probandi, al presumirse que todo acto en ejercicio de un derecho personal por una persona menor de edad que cuenta con el desarrollo, madurez y edad suficiente, se reputa realizado con discernimiento, intención y libertad.

De esta manera, quien alegue lo contrario, debería probarlo" (Minyersky, Herrera, 2006: 56).

Una salida menos conflictiva, que eluda un proceso casi anual, la intervención de multiplicidad de profesionales, la sobreexposición de una adolescente $\mathrm{y} / \mathrm{o}$ niña a un proceso judicial, y acorde con los principios fundantes de la LIG, en particular con la desjudicialización y la autodeterminación, hubiese sido que, en la situación bajo examen, el progenitor ausente de la joven trans sostenga y pruebe la incapacidad de ésta para ejercer su derecho personalísimo a la identidad de género como derecho humano (art. 75 inc. $22 \mathrm{CN}$, arts, 7, 8, 9, CDN; art. 18 de la Convención Americana sobre Derechos Humanos; art. 1, 2 y cc. LIG) y, en el peor de los casos, se llegue a los estrados de un juzgado para su determinación. El criterio rector podría ser el siguiente: el consentimiento de la joven trans, con el asentimiento de sus progenitores/as.

Otra interpretación posible hubiese sido que opere como condición solo la conformidad de uno/a de los/as progenitores/as o representantes legales a los efectos de dar trámite al pedido de rectificación del niño/a o adolescentes, teniendo en cuenta que aquellos/as son peticionarios/as en representación de la voluntad de éstos/as (Burgués, Navarro, 2016:154), cuestión ya saldada en la situación de J., teniendo en consideración la actitud tomada por su madre. Finalmente, queda pendiente pensar en la posibilidad de cuestionar la autoridad y/o legitimidad de un adulto -padre biológicoausente en la crianza y cuidado de una joven, solo presente para cuestionar derechos personalísimos, como el ejercicio del derecho a la identidad de género.

Finalmente, cabe mencionar cómo en el transcurso de la sentencia, no hubo mención a los Principios de Yokyakarta -que establecen directrices elementales para los Estados y actores sociales en materia de discriminación por orientación sexual e identidad de género- diagramados en Indonesia en el año 2006, en el marco de una reunión de especialistas y referentes en el campo de los derechos humanos, sexualidades y género, y que han sido referencias para el dictado de resoluciones y políticas públicas en el ámbito nacional e internacional. 


\section{A modo de cierre}

No es posible estandarizar el análisis como si fuese cualquier sujeto/a judicializado/a, más aún si tenemos en cuenta que "la(s) vulnerabilidad(es) de los cuerpos trans que se someten al sistema judicial en busca de un reconocimiento de parte del Estado en relación con su identidad y expresión genérica proviene de estar sujetados al mundo de los "otros" (Litardo, 2012:65).

Además, para abordar la situación de la joven trans no debe eludirse el principio de autonomía personal -más allá del principio de autonomía progresiva ya desarrollado- consagrado en el artículo 19 de la Carta Magna ${ }^{13}$; éste implica que:

siendo valiosa la libre elección individual de planes de vida y la adopción de ideales de excelencia humana, el Estado -y los demás individuos- no deben interferir en esa elección, limitándose a diseñar instituciones que faciliten la búsqueda de esos planes de vida y la satisfacción de los ideales de virtud que cada uno sustente e impidiendo la interferencia mutua en el curso de tal persecución” (Nino, 1989: $204-$ 205).

La LIG claramente es una política pública válida para el desarrollo de planes de vida y ciudadanía plena de adultos/as y niños/as y adolescentes travestis, transexuales y transgénero.

La edad mínima legal establecida para el ejercicio de derechos es un criterio rector que puede funcionar como principio, pero que no debe ser un impedimento automático, de acuerdo a los derechos y principios contemplados tanto en la CDN, como en la Ley 26.061 y el CCyCN. Esto, teniendo en cuenta que:

...las normas no son unívocas y que los jueces no son sus mecanismos aplicadores; que las primeras adquieren significación de acuerdo al modo en que son articuladas y relacionadas con los hechos relevantes del caso y que los segundos son sus intérpretes paradigmáticos a través de actos de constitución de sentido (performativos) que no son actos puramente individuales, sino inexorablemente sociales. Es decir, sobredeterminados por los intercambios simbólicos del proceso general de la interacción humana en un momento y lugar determinados (Cárcova, 2014: 16).

13 Constitución Nacional Artículo 19. Las acciones privadas de los hombres que de ningún modo ofendan al orden y a la moral pública, ni perjudiquen a un tercero, están sólo reservadas a Dios, y exentas de la autoridad de los magistrados. Ningún habitante de la Nación será obligado a hacer lo que no manda la ley, ni privado de lo que ella no prohíbe. 
Ya en 1986, la Corte de los Lores británica abría las puertas, en "Gillick vs. West Norfolk and Wisbech Area Health Authority"14 -luego conocida como "doctrina Gillick" o "Gillick Competent"-, a la posibilidad de que una persona que no alcance la edad mínima legal establecida para el ejercicio de derechos personalísimos, puede hacer uso de esta posibilidad por estar en condiciones de comprender su decisión por su grado de madurez.

No es posible concebir que, para el reconocimiento de la identidad de género autopercibida, sea necesaria, como surge de la sentencia analizada y de las intervenciones de los/as distintos/as profesionales, la presencia de "las crueldades tácticas que sustentan la identidad coherente" (Butler, 2008:173), es decir, cómo se exige -y sobrejerarquiza- una homogeneización y cohesión entre la identidad, la expresión de género y la sexualidad, de acuerdo a cánones normativos. Considero que no es posible pensar a la identidad de género como una suerte de formulario a completar, con ciertos requisitos, determinados y/o determinables por diferentes profesionales.

Inevitablemente al abordar -y judicializar- la situación de J., debemos remitirnos a la experiencia de Luana, una niña transgénero de -en su momento- 6 años, y la lucha de su madre, junto a distintos/as profesionales amigables, para el reconocimiento de su identidad de género autopercibida ${ }^{15}$. Si bien en esta situación, las aristas y complejidades fueron otras -como el rol de los medios de comunicación masivos, las decisiones de los organismos administrativos, la edad de la niña, etc., - la represión del deseo subjetivo y la sanción de la violación del mandato identitario estuvieron presentes 'desde afuera' al momento de la determinación y comprensión de las vivencias de una joven transgénero.

Repensar la sexualidad y el género "subvirtiendo los patrones de la reproducción, de la propiedad privada de los cuerpos y los placeres" (Grande, 2016: 63) es un camino arduo pero posible, si no asumimos roles policiales disciplinadores de la subjetividad de las personas -y, sobre todo, de niños, niñas y adolescentes- y tomamos distancia de las concepciones dominantes que imperan en el campo del derecho.

14 En este caso, Victoria Gillick, católica y madre de diez hijos/as, denunció a las autoridades sanitarias para impedir la aplicación de una resolución gubernamental que autorizaba la entrega de anticonceptivos a jóvenes menores de 16 años. Gillick quiso asegurarse, en uso de la "patria potestad", que ninguna de sus hijas/os reciban anticonceptivos, sin su consentimiento.

15 Para una mayor profundización sobre esta situación puede consultarse: Mansilla, Mónica Gabriela (2014) Yo nena, yo princesa. Luana, la niña que eligió su propio nombre, Los Polvorines: Universidad Nacional de General Sarmiento. 


\section{Bibliografía}

Abogadxs por los Derechos SeXuales (ABOSEX). "Publicamos la parte pertinente de la sentencia judicial que reconoce la identidad de género de una adolescente trans en provincia de Buenos Aires según la ley 26.743", Disponible en: <https://abosex.com.ar/2016/06/06/publicamos-la-parte-pertinente-de-la-sentenciajudicial-que-reconoce-la-identidad-de-genero-de-una-adolescente-trans-en-provinciade-buenos-aires-segun-la-ley-26-743/>

Asamblea General de las Naciones Unidas (20 de noviembre de 1989), Convención sobre de los Derechos del Niño [1989].

Beloff, M. (2011). "La traducción latinoamericana de la Convención sobre Derechos del Niño”, En: Flah, Lily R. (dir.). Los desafíos del derecho de familia en el siglo XXI. Buenos Aires: Errepar.

BEN, P. (2000). “Muéstrame tus genitales y te diré quién eres. El 'hermafroditismo' en la Argentina finisecular y de principios de siglo XX", En: Acha, O. y Halperín, P. (Comps.), Cuerpos, géneros e identidades. Estudios de Historias y Género en Argentina, Buenos Aires, Ediciones del Signo.

Berkins, L. (2008). "Un itinerario político del travestismo”, en Maffía, D. (Comp.), Sexualidades migrantes. Género y transgénero, Buenos Aires, Scarlett Press.

BERKINS, L. (2013). La gesta del nombre propio. Informe sobre la situación de la comunidad travesti en la Argentina, Buenos Aires: Ediciones Madres de Plaza de Mayo.

Burgués, M. B. y Navarro, E. M. (2016). "El nombre de los deseos”, en Paván, V. (Comp.) Niñez trans: experiencia de reconocimiento y derecho a la identidad, Los Polvorines, Universidad Nacional de General Sarmiento.

Butler, J. (2008). Cuerpos que importan: sobre los límites materiales y discursivos del "sexo", Buenos Aires, Paidós.

Cárcova, C. (2014). "El derecho y sus enlaces con la literatura y el cine", en La Letra y la ley. Estudios sobre derecho y literatura, Buenos Aires, Infojus.

Ciolli, M. L. (2012). "Ley de identidad de género", en Suplemento Especial Identidad de género - Muerte digna, Buenos Aires, La Ley, p.11-12.

CuriA, D. (2016). "Niñxs primero", En: Página 12, Suplemento Soy. Disponible en:https://www.pagina12.com.ar/diario/suplementos/soy/1-4531-2016-05-08.html.

Congreso de la Nación Argentina (28 de septiembre de 2005), Ley de Protección Integral de los Derechos de los Niños, Niñas y Adolescentes [Ley 26.061 de 2005].Disponible en: $<$ http://servicios.infoleg.gob.ar/infolegInternet/anexos/110000114999/110778/norma.htm >.

Congreso de la Nación Argentina (9 de mayo de 2012), Ley de Identidad de Género [Ley 26.743 de 2012]. Disponible en: < http://servicios.infoleg.gob.ar/infolegInternet/anexos/195000199999/197860/norma.htm>. 
Congreso de la Nación Argentina (1 de octubre de 2014), Código Civil y Comercial de la Nación [Ley 26.994 de 2014]. Disponible en: < http://servicios.infoleg.gob.ar/infolegInternet/verNorma.do?id=235975>.

DuARTE, K. (2006). Discursos de Resistencias Juveniles en Sociedades Adultocéntricas, Santiago de Chile, DEI.

FERNÁNDEZ, S. (2012). "La realización del proyecto de vida autorreferencial. Los principios de autonomía y des judicialización”, en Suplemento Especial Identidad de género - Muerte digna, Buenos Aires, La Ley.

Grande, A. (2016). "Nuestra pequeña Lulú”, en Pavan, V. (Comp.) Niñez trans: experiencia de reconocimiento y derecho a la identidad, Universidad Nacional de General Sarmiento, Los Polvorines.

Güemureman, S. y Macri, M. R. (2013). "La configuración del campo de estudios sobre juventud, adolescencia e infancia en Argentina a partir de la recuperación de la democracia”, en Revista de Ciencias Sociales, Dossier Itinerarios de investigación, Mar del Plata, Sudamérica.

Jaramillo, I. C. (2008). "Capítulo tres Familia”, en Motta, C. y Sáez, M. (Comps.), La mirada de los jueces. Género en la jurisprudencia latinoamericana. Tomo 1, Siglo del Hombre Editores, Bogotá.

LiTARDO, E. (2012). "Derecho e identidad trans. Escupamos sobre Júpiter”, en Revista de la Facultad de Ciencias Sociales $N^{\circ}$ 81, Universidad de Buenos Aires, Buenos Aires.

MeCciA, E. (2012). "Imaginación jurídica y experiencias trans", en Barrancos, D. (Comp.), Moral, géneros y sexualidades en Argentina, Katz, Buenos Aires, en prensa.

MedinA, G. (2012). "Comentario exegético a la ley de identidad de género", En: Suplemento Especial Identidad de género - Muerte digna, Buenos Aires, La Ley.

Minyersky, N., Herrera, M. (2006). "Autonomía, capacidad y participación a la luz de la ley 26.061", en García Méndez, E. (comp.) Protección Integral de Derechos de Niñas, Niños y Adolescentes. Análisis de la ley 26.061, Editores del Puerto, Buenos Aires.

Orozco, A. y LA Fuente S. (2014). "Economía y (trans) feminismo. Retazos de un encuentro", en Transfeminismos. Epistemes, fricciones y flujos, Navarra, Txalaparta.

Pecheny, M. (2008) "Introducción. Investigar sobre sujetos sexuales", en Pecheny, M., Figari, D., y Jones, D., (Comps.) Todo sexo es político. Estudios sobre las sexualidades en Argentina, Buenos Aires: Libros del Zorzal.

Pérez Cazenave, L. (2017). "Cambio de paradigma legal, actuación del Estado y estigmatización de lxs jóvenes”, en Derechos en Acción (ReDeA), N², La Plata, Universidad Nacional de La Plata, p.341-354. Disponible en: <http://revistas.unlp.edu.ar/ReDeA/article/view/2856>. 